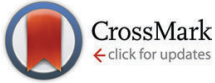

Cite this: J. Mater. Chem. C, 2016 4, 2178

Received 21st October 2015, Accepted 13th February 2016

DOI: $10.1039 / c 5 t c 03448 e$

www.rsc.org/MaterialsC

\section{Real-time evaluation of thin film drying kinetics using an advanced, multi-probe optical setup $\dagger$}

\author{
Nusret S. Güldal, ${ }^{* a}$ Thaer Kassar, ${ }^{b}$ Marvin Berlinghof, ${ }^{b}$ Tayebeh Ameri, ${ }^{a}$ \\ Andres Osvet, ${ }^{a}$ Roberto Pacios, ${ }^{c}$ Giovanni Li Destri, ${ }^{d}$ Tobias Unruh ${ }^{\star b}$ and \\ Christoph J. Brabec ${ }^{a}$
}

\begin{abstract}
Solution-processed organic photovoltaic devices are advantageous due to their low-cost large area manufacturing techniques, such as slot-die coating, gravure printing and roll-to-roll coating. The final microstructure of a polymer:fullerene bulk-heterojunction $(\mathrm{BHJ})$ film is a fine interplay between solution thermodynamics (e.g. solubility, miscibility...) and kinetics (e.g. solvent evaporation, polymer ordering, phase separation...) during the drying process. In order to design better performing organic photovoltaic devices, gaining knowledge over the drying properties of polymer:fullerene thin films is essential. A novel in situ thin film drying characterization chamber, equipped with white-light reflectometry, laser light scattering and photoluminescence, is presented in combination with grazing-incidence $X$-ray diffraction on two different polymer:fullerene bulk heterojunctions based on poly-(3-hexylthiophene2,5-diyl) (P3HT) and polythieno[3,2b]thiophene-diketopyrrolopyrrole-co-thiophene (DPP-TT-T) polymers. With photoluminescence applied for the first time as an in situ method for such drying studies, these single-chamber measurements track the fine interplay between thermodynamics and kinetics of thin film drying and provide invaluable information on solution behavior and microstructure formation.
\end{abstract}

\section{Introduction}

Solution-processed organic electronics benefit from their lowcost large area manufacturing techniques, such as slot-die coating, gravure printing and roll-to-roll coating. ${ }^{1,2}$ Organic semiconductors in the form of thin films are extremely sensitive to processing conditions, which lead to different microstructures, subsequently affecting performance. An optimized polymer:fullerene bulk-heterojunction ( $\mathrm{BHJ})$ is a finely mixed, yet phase separated network, consisting of a conjugated polymer as a donor and a fullerene-derivative as an acceptor. It has a delicate morphology that is affected by the solvent choice, coating conditions and post-treatments, such as thermal annealing and solvent annealing. ${ }^{3-6}$ The final microstructure of a $\mathrm{BHJ}$ film is determined by the interplay between thermodynamics (e.g. solubility, miscibility...) and kinetics (e.g. solvent evaporation,

\footnotetext{
${ }^{a}$ Materials for Energy Technology and Electronics, Friedrich-Alexander University Erlangen-Nürnberg, Martensstrasse 7, 91058, Erlangen, Germany. E-mail: nusret.s.guldal@fau.de

${ }^{b}$ Chair for Crystallography and Structural Physics, Friedrich-Alexander University Erlangen-Nürnberg, Staudtstrasse 3, 91058, Erlangen, Germany

${ }^{c}$ IK4-IKERLAN, Paseo J. M. Arizmendiarrieta 2, 20500, Arrasate-Mondragon, Spain

${ }^{d}$ European Synchrotron Radiation Facility, 71 Avenue des Martyrs, 38000, Grenoble, France

$\dagger$ Electronic supplementary information (ESI) available. See DOI: 10.1039/ c5tc03448e
}

polymer ordering, phase separation...) during the drying process. ${ }^{7,8}$ Therefore, gaining knowledge over the drying properties of polymer/fullerene thin films and correlating this information with the performance of lab-scale $\mathrm{BHJs}$ are essential in order to design better performing organic photovoltaic devices and transfer the manufacture protocols to large scale production.

Over the past decade, a considerable amount of in situ characterization methods has been used to study drying dynamics and microstructure evolution of BHJs. ${ }^{9}$ One of the most popular methods is white-light/laser reflectometry, using which the evolution of drying film thickness can be determined. ${ }^{7,8,10-18}$ Instead of reflectometry, some investigations have employed spectroscopic ellipsometry (SE), which is more sensitive to sub-nanometer regions, allowing the user to probe final stages of thin film drying. ${ }^{19-21}$ Additionally, laser light and $\mathrm{X}$-ray scattering techniques are used either as stand-alone methods or in combination with other optical methods. While laser light scattering has been used as a tool to investigate liquid-liquid phase separation in organic blends, grazingincidence X-ray scattering, with its wide (GIWAXS) and small angle (GISAXS) forms, has become a very powerful technique to investigate periodic ordering and domain formation in organic thin films, respectively. ${ }^{10,12,18,22,23}$

So far, spin coating has been one of the most favoured casting methods to study in situ morphology formation due to its importance and prevalence in lab-scale organic film fabrication. ${ }^{10,16-19,24}$ 
In spite of the invaluable information presented until now on the matter, the applicability of the results to build a general road map for solvent and drying effects on microstructure formation is rather limited since the drying on a spin coater is not comparable to the one on any printing machine. Thus, in the last few years in situ measurements from blading and bar spreading methods became as popular as spin coating due to their similarities to industrial-scale printing methods.,8,11-15,20,21,23,25 Nevertheless, regardless of whether spin coating is selected or doctor blading, in situ characterization methods are mostly utilized to get deeper insight into the morphology and correlate this information with device performance. There is a very limited amount of reports which studied the precision of solution formulation and drying conditions on the thermodynamics and kinetics of the $\mathrm{BHJ}$ thin film drying, thus the current knowledge is still far from building a road map for better solution formulations and drying conditions, which could be transferred to large-scale processing for further optimizations.

In this work, we present a very portable and flexible in situ measurement setup, which is a fully automated miniature doctor blade with a controlled heating system and atmosphere. This setup was built in a way that the user can control the coating and drying conditions very precisely, thus it enables full characterization of the drying behavior of a thin film without the need of alter or switch setups. To achieve full characterization within a single setup, it is equipped with white-light reflectometry (WLR), laser light scattering (LS) and photoluminescence (PL). Although few reports exist on anti-graffiti coatings and hole injection layers for organic light-emitting diodes, to our knowledge, this is the first time in situ PL is used as a real-time measurement technique to observe the drying of BHJs. ${ }^{26,27}$ Since PL is a very sensitive method to probe thermodynamic changes in a solution containing a luminescent material, utilizing PL in combination with other methods makes this setup one of its kind, as it allows for a much deeper insight into the fine interplay of solution thermodynamics and drying kinetics. Thus, the user is able to optimize solution formulations and drying conditions for a better morphology before device fabrication.

The setup (Fig. 1) was built as a chamber with a volume of $38 \times 17 \times 9 \mathrm{~cm}^{3}$ (an atmospheric volume of around $3 \mathrm{~L}$ ) and optical components on top. It measures a spectrum (or data point) as fast as every $40 \mathrm{~ms}$ with a doctor blade speed range of 1-60 $\mathrm{mm} \mathrm{s}^{-1}$, a fine adjustable heating stage up to $180{ }^{\circ} \mathrm{C}$ and a controlled atmosphere which can be saturated with desired solvent, even at elevated temperatures. We demonstrate this portable setup under atmosphere control using WLR, LS, PL and grazing-incidence X-ray diffraction (GIXD) combined measurements to probe two different drying behaviors (homogeneous and heterogeneous) with two different polymer:fullerene blends, based on poly-(3-hexylthiophene-2,5-diyl) (P3HT) and poly-thieno[3,2b]thiophene-diketopyrrolopyrrole-co-thiophene (DPP-TT-T), each mixed with [6,6]-phenyl C61-butyric acid methylester (PCBM) (Fig. S1, ESI $\dagger$ ). From WLR measurements, a drying curve is established to monitor thickness evolution. For BHJ systems, LS informs on the phase separation, and PL allows the tracking of the molecular self-assembly during drying. These measurements, combined with the polymer ordering information achieved from GIXD measurements, reveal the mechanism and interplay between solubility, gelation, polymer agglomeration, chain aggregation, ordering and phase separation, which later will be important for understanding the factors affecting the drying and microstructure formation of BHJs for organic photovoltaics.

\section{Materials and methods}

\section{Materials and solutions}

Regioregular P3HT was purchased from Merck $\left(M_{\mathrm{w}}=65 \mathrm{kDa}\right)$, DPP-TT-T was provided by Solvay and $\mathrm{PC}_{60} \mathrm{BM}(99.5 \%)$ was purchased from Solenne BV. All materials were used as received. The $\mathrm{P} 3 \mathrm{HT}$ : $\mathrm{PCBM}$ solution was prepared by dissolving P3HT and PCBM in 1,2-dichlorobenzene (99\%) from Sigma-Aldrich with $1: 1 \mathrm{wt} \%$ and a total concentration of $22.5 \mathrm{mg} \mathrm{mL}{ }^{-1}$. Pristine P3HT solution was prepared in the same solvent at a concentration of $11.25 \mathrm{mg} \mathrm{mL}^{-1}$. The DPP-TT-T: PCBM solution was prepared by dissolving DPP-TT-T and PCBM in a solvent mixture with $1: 2 \mathrm{wt} \%$ and at a total concentration of $15 \mathrm{mg} \mathrm{mL}^{-1}$. The solvent mixture was from $o$-xylene (97\%) and mesitylene (98\%) from SigmaAldrich, with $7: 3 \mathrm{v} / \mathrm{v}$. The pristine DPP-TT-T solution was prepared
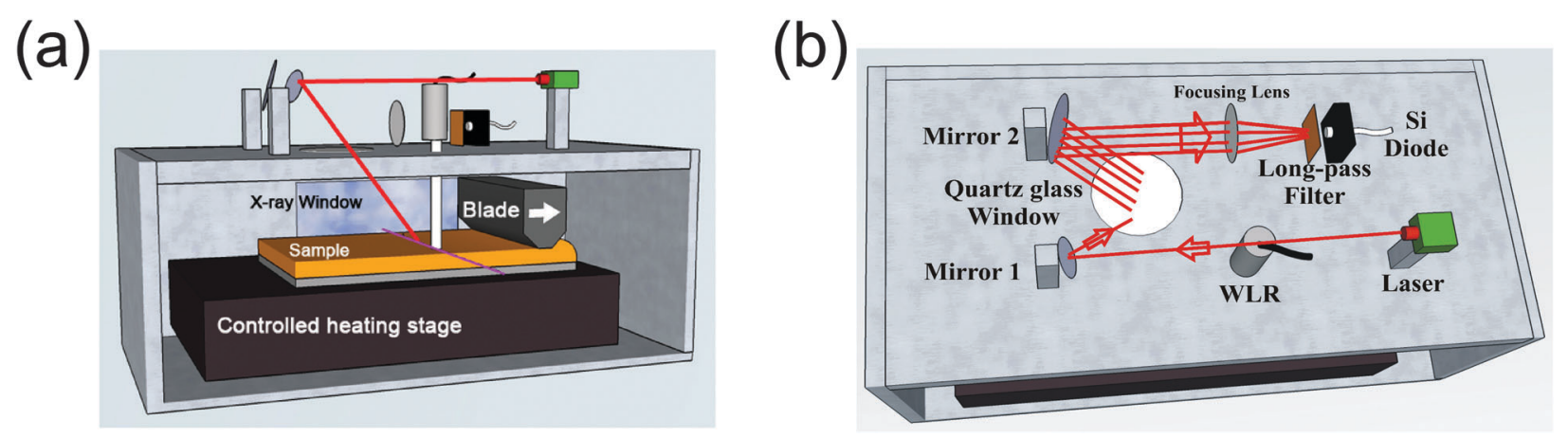

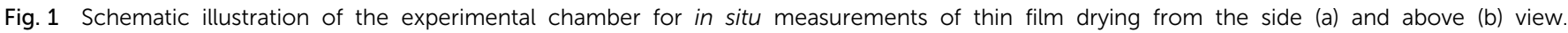

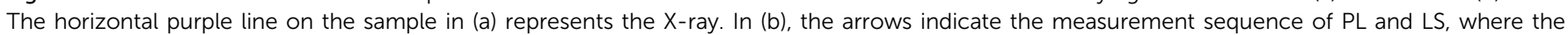
laser, Si diode and long-pass filter are changeable depending on the experimental conditions. 
in the same solution mixture at concentrations of 3.75, 5, 7.5 and $15 \mathrm{mg} \mathrm{mL}{ }^{-1}$. Another pristine DPP-TT-T solution was prepared in only $o$-xylene at $5 \mathrm{mg} \mathrm{mL}^{-1}$. All solutions were stirred at $65{ }^{\circ} \mathrm{C}$. Silicon wafers, purchased from Silicon Materials with natural $\mathrm{SiO}_{2}$, were cut into rectangular pieces $(20 \times 80 \mathrm{~mm})$, followed by mechanical cleaning with soapy de-ionized water, rinsing with de-ionized water, and ultra-sonication with acetone and then with iso-propanol. The substrates were dried with nitrogen and kept in a desiccator to avoid any humidity effects on the surface.

\section{Steady-state measurements}

Absorption spectra of the DPP-TT-T:PCBM solution and the film were recorded in a quartz cuvette and by blading on a microscope slide, respectively, using a Perkin Elmer Lambda $950 \mathrm{UV} / \mathrm{VIS} / \mathrm{NIR}$ spectrophotometer. Steady-state photoluminescence measurements were conducted using a custom-made spectrometer equipped with a $450 \mathrm{~nm}$ laser and a Horiba Symphony InGaAs detector. PL spectra were corrected with respect to optical density and detector sensitivity. Height profile measurements were conducted using a Tencor Alphastep D-500 Profilometer. Solubility measurements were conducted at $25{ }^{\circ} \mathrm{C}$ and $60{ }^{\circ} \mathrm{C}$, as explained in detail elsewhere. ${ }^{28}$ Atomic force microscopy (NT-MDT Solver Nano) for pristine P3HT was conducted on a Si wafer.

\section{In situ measurements}

White-light reflectometry was performed using a Filmetrics F20-UV and a fiber light source, L10290, from Hamamatsu. The integration time of the spectrometer was set to $250 \mathrm{~ms}$. Stingray lasers from Coherent Inc. with 516 and $783 \mathrm{~nm}$ wavelengths were used for laser light scattering and photoluminescence measurements. Lasers were triggered with a universal trigger with $0.25 \mathrm{~ms}$. $630 \mathrm{~nm}$ and $850 \mathrm{~nm}$ long-pass filters (RG630 and RG850, respectively, from Schott) were used for photoluminescence measurements to filter out the laser light. The optical geometry used for PL and LS measurements is as follows: the laser beam had an incidence angle of $34^{\circ}$ from the normal and directed in the direction of the blade movement, on the substrate. The polarization (E vector) was in the plane of incidence. The axis of the detection path was inclined from the plane of incidence by $23^{\circ}$ (azimuth angle); the polar angle was $37^{\circ}$. The numerical aperture of the polarizationinsensitive collection optics is 0.13 . The signal from laser light scattering and photoluminescence was collected using a silicon diode with an integrated transimpedance amplifier (Roithner LaserTechnik GmbH). The collected signal was then processed using a Signal Recovery dual-phase lock-in amplifier (model 7265) with a time constant of $200 \mathrm{~ms}$. In situ spectral photoluminescence measurements were carried out by replacing the Si-diode with a fiber UV-Vis spectrometer (Avantes Sensline), with the integration time set to 1 second. Especially for DPP-TT-T: PCBM samples, the whole PL spectrum could not be resolved using the UV-Vis spectrometer. Nevertheless, it was sufficient for tracking PL changes during drying. For LS and PL measurements, it should be noted that the top chamber wall carrying the optical components is black-anodized in order to avoid any reflections or scattering caused by the light scattered/reflected from the drying layer or Si wafer. The in situ GIXD measurements were conducted at the Beamline ID10 at European Synchrotron Radiation Facility (ESRF) in Grenoble, France, using a wavelength of 1.24 Å. As also mentioned in the main text, the WLR measurement spot and the LS/PL measurement spot are on the X-ray line, and they are separated by $2 \mathrm{~mm}$ from each other.

The chamber was implemented on the synchrotron sample stage with all the optical parts on top, including an automated solution injection system. X-ray windows (Nalophan) used for the blading chamber were purchased from Kalle. A 2D Pilatus $300 \mathrm{~K}$ detector from Dectris was used with a frame time of $1 \mathrm{~s}$. The sample detector distance was fixed at $170.33 \mathrm{~mm}$ after the calibration with silver behenate and the incident X-ray angle was set to $0.16^{\circ}$, which was below the critical angle of the Si substrates. All drying experiments were conducted at $60{ }^{\circ} \mathrm{C}$ under a dry helium atmosphere in stagnation. The temperature was regulated at $60{ }^{\circ} \mathrm{C}$ very precisely for the whole setup (chamber walls, substrate stage and He atmosphere). Before the chamber was filled with $\mathrm{He}$, He gas was heated up to $60{ }^{\circ} \mathrm{C}$ with a self-made gas heating system. The gas pipeline carrying warm He gas between the gas heating system and the drying chamber was additionally heated up to $60{ }^{\circ} \mathrm{C}$ and properly isolated in order to avoid temperature losses. The gas outlet of the chamber left open connected directly to a gas bubbler filled with ethanol in order to avoid overpressure in the chamber. Hence, all experiments were conducted under atmospheric pressure, and any condensation in the chamber was avoided. A total of $120 \mu \mathrm{L}$ of solution was bladed on a Si substrate. Measurements were started right after the applicator passed the X-ray windows and lasted for $3 \mathrm{~min}$. In order to avoid radiation damage, the blading chamber was moved horizontally $55.5 \mu \mathrm{m}$ per frame, in total $10 \mathrm{~mm}$. Since the samples were always bladed with a $15 \mathrm{~mm} \mathrm{~s}^{-1}$ blading speed, two consecutive frames had a $3.7 \mathrm{~ms}$ drying time difference. For a complete drying experiment, there was a $0.67 \mathrm{~s}$ drying time difference between the first and last frame, which is less than our time resolution. Nevertheless, this difference was corrected between optical and scattering measurements. Lastly, the crystal size and lamellar packing were calculated as described elsewhere. ${ }^{29}$

It is important to note that for measurements including synchrotron studies, a second blading chamber was used which has exactly the same size, volume, blading mechanism and temperature and speed precision as the chamber described in the text. This chamber is slightly modified for synchrotron usage: (1) it includes X-ray windows, and (2) the blading mechanism is operated with pressurized air in order to avoid vibrations. For both polymer:fullerene systems, LS was measured separately as PL and LS need the same measurement tools. Nevertheless, LS measurements were conducted together with WLR, thus a drying comparison with the rest of the measurements was possible.

\section{Results and discussion}

\section{Homogeneous drying}

P3HT : PCBM solutions (22.5 $\left.\mathrm{mg} \mathrm{mL}^{-1}\right)$ were prepared with $1: 1$ $\mathrm{w} / \mathrm{w}$ in ortho-dichlorobenzene (o-DCB) while DPP-TT-T: PCBM 
solutions (15 $\mathrm{mg} \mathrm{mL}^{-1}$ ) were prepared with a $1: 2$ weight ratio in the $o$-xylene and mesitylene mixture $(7: 3 \mathrm{v} / \mathrm{v})$. Solutions were injected using an automatized injection system on pre-cleaned silicon substrates and coated at $60{ }^{\circ} \mathrm{C}$ under a dry helium atmosphere. All measurements were initiated at the same time, immediately following the coating, with the first spectrum taken at the 0th second of drying. Two sets of data were collected for each blend: (1) synchrotron studies were conducted in combination with WLR and PL (Fig. S2, ESI $\dagger$ ), and (2) LS measurements were done under identical drying conditions, in combination with WLR. To be able to compare LS data with the first set of data, thickness plots for each polymer:fullerene blend include both drying curves calculated from WLR measured for each dataset. This ensures that although LS was measured separately, we were able to reproduce the drying very precisely, thus LS data could be included to the other measurements. In a typical drying curve of a P3HT:PCBM film, the film thickness is calculated from the interference fringes of the reflectance spectrum measured by WLR (Fig. 2a). The principles of the thickness calculation are based on the fringe counting method, as it is explained in detail elsewhere and in the ESI, $\dagger$ Section 2. ${ }^{8,30}$
The P3HT:PCBM film was dried with a predominantly constant evaporation rate (evaporation-dominated drying) followed by a period of slow drying. The latter occurred in a relatively thin region of less than two microns, where the molecular arrangements during film formation caused major changes in the optical constants, thus the loss of interference. ${ }^{21}$ The drying period below $2 \mu \mathrm{m}$ cannot be resolved quantitatively by our thickness calculation approach. Nevertheless, it is observed that this final phase of film formation had a slightly slower drying behavior, as the final WLR signal deviated from the extrapolation of the linear behavior. This slow drying region of the P3HT:PCBM system was observed previously, and it was attributed to diffusion-dominated drying due to the residual solvents. ${ }^{8}$ At the end of this slow drying phase, the final film thickness was determined as $234 \mathrm{~nm}$.

The time evolution of the LS signal is plotted in Fig. $2 \mathrm{~b}$. The blue line indicates the onset of the LS signal, approximately at the 60th second of drying. The inset in Fig. 2b shows that the onset is at the 58th second, which was challenging to observe due to signal fluctuations. This sudden increase in LS intensity was a sign of the first stages of film formation, either induced
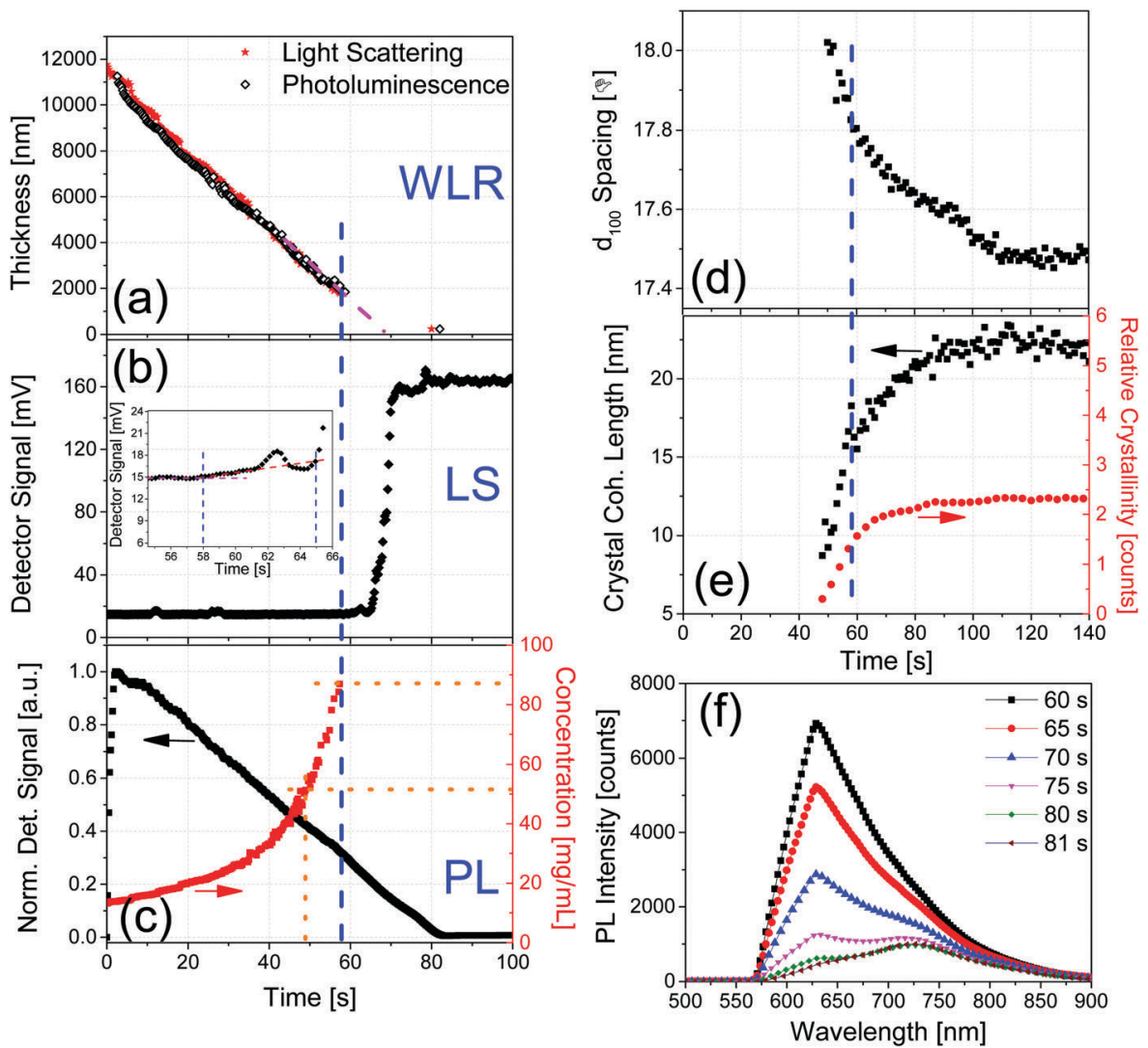

Fig. 2 Time evolution of P3HT:PCBM drying measured by WLR (a), LS (b), PL (c), GIXD (d \& e) and spectral PL (f). Drying curves for LS (red star) and PL (black circle) are plotted together in (a) for comparison. The blue dashed line in (a)-(e) represents the onset of LS. The inset figure in (b) zooms in the LS data between the 55th and 66th seconds. Dashed lines are used in this inset to clarify the LS onset, which is the 58th second of the drying. Panel (c) contains the partial P3HT concentration profile calculated from WLR data measured together with PL. Orange dotted lines in panel (c) represent the concentration values for the onsets of crystallization (48th second) and LS (58th second). Panels (d) and (e) show the lamellar packing, the crystal coherence length in the $q_{z}$ direction and relative crystallinity (integrated (100) pole figure) calculated from the in situ GIXD measurements. Spectral PL results in (f) are plotted from the 60th second (black box) on, and the PL spectrum loses intensity and red shifts until the 81 st second (brown angle bracket) of drying. Since spectral PL measurements were conducted separately, mismatch in timing might be present. 
by the blend going through a spinodal demixing of components during drying (i.e., liquid-liquid phase separation) or by crystallization (i.e., solid-liquid phase separation), which are both wavelength-sensitive. ${ }^{10,18,31}$ Our findings suggest that the origin of the LS signal comes from a late-stage solid-liquid phase separation between fullerene- and polymer-rich phases as the pristine $\mathrm{P} 3 \mathrm{HT}$ gives a similar signal, but with a significantly reduced intensity (Fig. S3a, ESI $\dagger$ ). In order to rule out the surface roughness as the origin of the LS signal for pristine P3HT, we have additionally conducted AFM measurements on the pristine P3HT layer (ESI, $\dagger$ Section1). Interestingly, GIXD data on P3HT:PCBM show that the formation of P3HT crystals started slightly earlier (approximately the 50th second of drying) than the onset of the LS signal (Fig. 2e). This indicates that the nucleation and crystal formation of the polymer may be the driving force for solid-liquid phase separation, which is supported by the findings, reported earlier on the same system. ${ }^{13,16}$ Hence, the results indicate that the LS signal is a consequence of the coarsening of polymer- and fullerene-rich phases, i.e. causing surface modulation between the phases, especially with sizes in the order of the excitation wavelength.

The integrated PL behavior during drying is plotted in Fig. 2c for P3HT:PCBM. The initial increase was caused by a slightly large lock-in integration time constant (200 ms) which required approximately one second of data reading before delivering a stabilized output. The PL signal started to decrease immediately after this initial period, with a linear decay until the layer was completely dried. There are several PL quenching mechanisms for fluorescent conjugated semiconductors: intrachain defects, impurities/dopants, photochemical oxidation, concentration quenching, aggregation quenching leading to interchain species (e.g., excimers, exciplexes, polaron pairs), as well as an efficient photo-induced charge transfer to a quencher. $^{32,33}$ In theory and practice, it was shown that PL decay kinetics is a function of concentration. ${ }^{33}$ At sufficiently diluted concentrations, the polymer chains do not 'see' each other and the PL signal remains constant during the drying (Fig. S4, ESI $\dagger$ ). However, the concentrations that we present here are high enough to observe concentration quenching until the polymer concentration reaches the critical aggregation concentration (Fig. S5, ESI $\dagger$ ). At a certain time point during drying, the relative increase of polymer concentration starts to promote polymer chain aggregation, thus aggregation-induced PL quenching fastens the PL decay over time alongside concentration quenching.

Comparing the kinetics of the pristine P3HT solution, Fig. 2c shows mainly a competition between a charge transfer to the quencher, PCBM, concentration quenching and aggregation quenching. Since there are no significant patterns in the PL signal at the onset of the LS signal, spectral PL was measured under the same drying conditions (Fig. 2f). The emission spectra were cut off by a $630 \mathrm{~nm}$ long-pass filter; hence the data below $630 \mathrm{~nm}$ are not representative for P3HT emission. A spectral red shift and the evolution of a shoulder at approximately $750 \mathrm{~nm}$ temporally correlate with the onset of aggregation, as well as with the $\mathrm{P} 3 \mathrm{HT}$ crystal formation, and thus were assigned to the formation of P3HT aggregates. These features indicate energy transfer being enhanced due to increased $\pi-\pi$ interactions, forming nanoscale aggregates, which shifts the PL spectrum to longer wavelengths. ${ }^{32}$ This also suggests that the PL behavior between the 60th and 80th seconds of drying is dominated not only by the concentration quenching but also by aggregation quenching, which together with fullerene quenching cause a linear behavior in the integrated signal.

P3HT crystal growth continued even after the reflection spectrum stopped changing and phase separation was completed (Fig. 2d and e). The formation and growth of crystals stabilized at approximately the 100th second with a size and $d_{100}$-spacing of $23 \mathrm{~nm}$ and $17.5 \AA$, respectively. This indicates that P3HT experienced a prolonged self-organization after the period of slow drying (i.e., from the 80th second on), possibly due to the increased molecular kinetics at $60{ }^{\circ} \mathrm{C}$. Fully dried films after the 100th second exhibited a fairly high order with the edge-on (h00) series, at $q_{\mathrm{z}}=0.36 \AA^{-1}, 0.7 \AA^{-1}$ and $1.1 \AA^{-1}$ for (100), (200) and (300), respectively (Fig. S6a, ESI $\dagger$ ). ${ }^{12}$

\section{Heterogeneous drying}

In order to test the setup as a powerful characterization tool for drying and morphology evolution, we measured DPP-TT-T: PCBM blend as a second prototype composite relevant to photovoltaic devices. DPP-TT-T is a push-pull type conjugated polymer, well known for its planarity and rigidity due to two thieno $[3,2 b]$ thiophene moieties that increase the conjugation length, promoting chain aggregation. ${ }^{34,35}$ DPP-TT-T has been studied extensively, especially in transistors, due to its good ambipolarity and hole mobility above $1 \mathrm{~cm}^{2} \mathrm{~V}^{-1} \mathrm{~s}^{-1} \cdot{ }^{36}$ Lately, the very same polymer showed a power conversion efficiency of $6.6 \%$ in spin coated organic photovoltaic devices via molecular weight tuning. ${ }^{37}$

The normalized steady-state absorption spectra of pristine DPP-TT-T in solution and as a film showed no significant spectral shift except a broadening of the signal (Fig. S7a, ESI $\dagger$ ). Steady-state PL measurements of pristine DPP-TT-T showed two peaks in the UV-Vis and NIR regions (Fig. S7b, ESI $\dagger$ ). The NIR emission represents the singlet emission, while the UV-Vis emission represents an energetically higher lying excited state of DPP-TT-T. The high-energy emission at $680 \mathrm{~nm}$ increased with concentration up to $3.75 \mathrm{mg} \mathrm{mL}^{-1}$, which was completely quenched at $5 \mathrm{mg} \mathrm{mL}^{-1}$, while the singlet emission within 900$1250 \mathrm{~nm}$ continuously increased with concentration. The quenching effect at $680 \mathrm{~nm}$ at $5 \mathrm{mg} \mathrm{mL}^{-1}$ indicates a sufficient number of polymer/polymer interfacial contacts which quench the higher energy excited state completely. Since DPP-TT-T: PCBM drying experiments were conducted with a polymer concentration of $5 \mathrm{mg} \mathrm{mL} \mathrm{mL}^{-1}$, this suggests that DPP-TT-T already has some sort of an ordering or aggregation in solution. The drying curve shows that composites of DPP-TT-T:PCBM in the $o$-xylene:mesitylene solvent mixture dried with a constant drying rate (Fig. 3a). Although we expected to have residual amounts of mesitylene in the semi-dry films which could cause a slower drying rate towards the end, it was not observed. Unlike 

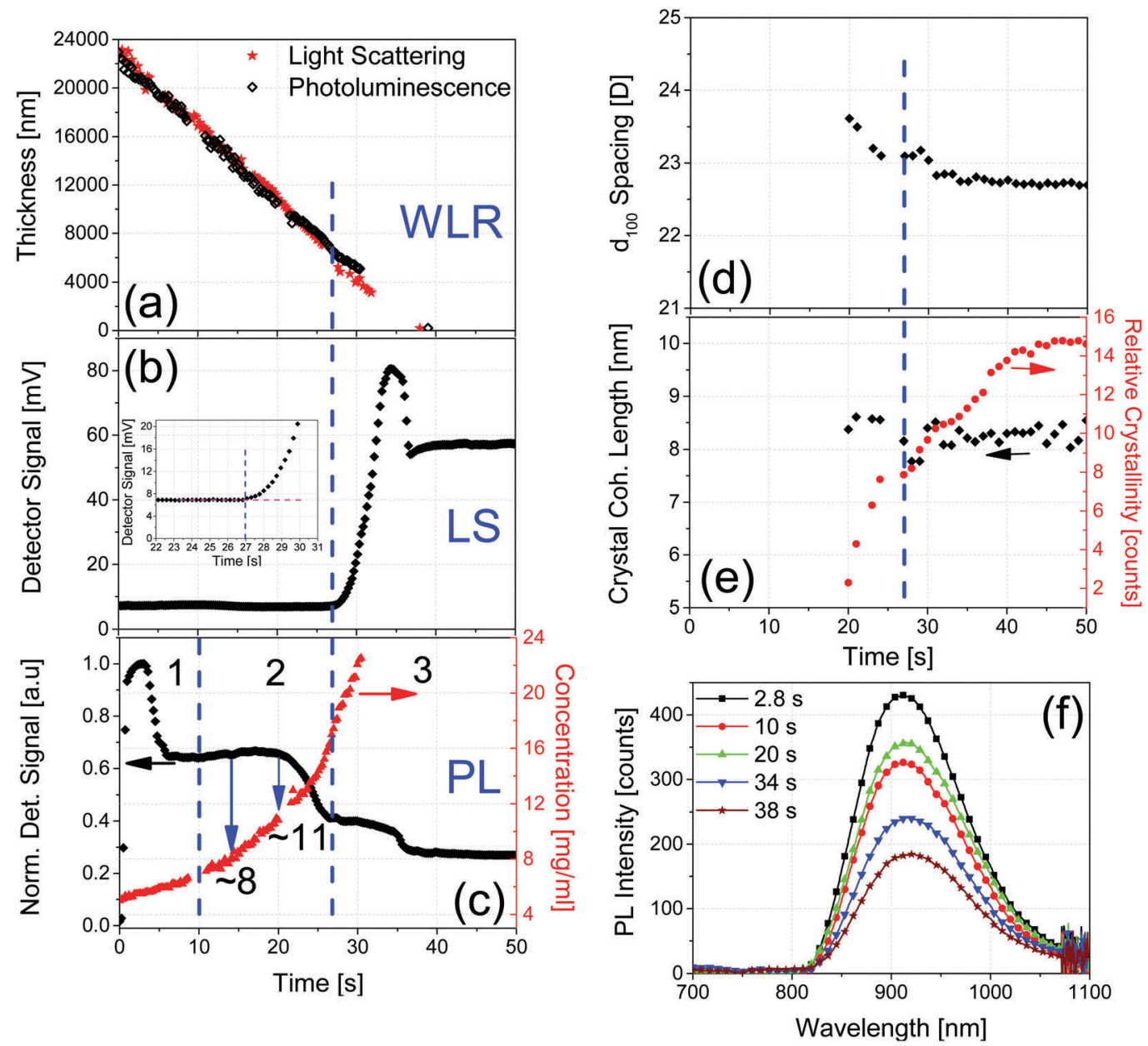

Fig. 3 Time evolution of DPP-TT-T:PCBM drying was measured by WLR (a), LS (b), PL/partial polymer concentration profile (c), GIXD (d \& e), and spectral $\mathrm{PL}$ (f). The long dashed line in (a)-(c) represents the onset of the LS signal. Dashed lines separate in situ PL in (c) into three regions, labelled as 1,2 and 3. Vertical blue arrows in (c) represent the polymer concentration at the corresponding PL value. Horizontal arrows in (c) show the corresponding $y$-axis for each plot. Spectral PL results in (f) are plotted from the 2.8th second (black box) on, and the PL spectrum loses intensity until the 38th second (brown star) of drying. Since spectral PL measurements were conducted separately, a minute mismatch in timing might be present.

the P3HT:PCBM system, the final WLR signal for DPP-TT-T:PCBM followed the extrapolation of the linear behaviour. This could be due to the interaction between non-volatile components in the solution with mesitylene, allowing mesitylene to diffuse to the film surface for further evaporation without any restriction. Therefore, it was observed that the thickness evolution continued linearly until the end of the drying, without any slow drying regime, and the final film thickness was measured as $389 \mathrm{~nm}$.

A large scale phase separation was observed again at the very late stage of drying (Fig. 3b). The onset of crystal formation and ordering was observed ahead of the large-scale phase separation and film formation (Fig. 3d and e), which was also observed for P3HT:PCBM. The origin of the LS signal, however, may differ for the DPP-TT-T:PCBM composites; as the pristine polymer dried very homogenously and barely showed any LS signal (Fig. S3b, ESI $\dagger$ ), the LS signal in Fig. 3b might have originated primarily from fullerene-rich domains. Most interestingly, we did not observe a phase of well-expressed crystal growth at the late stages of drying, and the final crystal formation ended with the onset of the LS signal (Fig. 3e). This result supports the fact that the $o$-xylene:mesitylene mixture allows only negligible ordering of crystals at the semi-dry state of these films.

The PL behavior of DPP-TT-T:PCBM was very different from the one observed for P3HT:PCBM during drying (Fig. 3c). The very first sharp decrease of the PL signal was due to the coffeering effect (region 1, Fig. 3c). Depending on the surface energy, solvent evaporation could be faster at the edges than in the middle of the coating due to the larger surface area per volume at the edge. The contact line at the liquid/substrate interface gets pinned and the surface tension introduces a capillary flow from the center of the film towards the edges. This capillary flow is causing a solute transfer via advection and results in thinner films at the center than at the edges. ${ }^{38}$ Unlike the P3HT:PCBM system, a coffee-ring effect was observed for DPP-TT-T:PCBM coatings (Fig. S8, ESI $\dagger$ ). Such a drying pattern has a fairly minor effect on reflection data and thickness 
calculations; however, PL is significantly more sensitive to the amount of luminescent material under the measurement spot. Since the laser light for PL measurements was focused on the center of the film to avoid possible drying effects, the initial early phase decrease in the PL signal was attributed to the capillary flow transporting the polymer towards the edges, away from the measurement spot.

A slightly increasing trend in the PL signal was observed (region 2, Fig. 3c); however, any spectral shifts during this drying phase were ruled out by the spectral PL measurements (Fig. 3f). We do not want to overemphasize the slight trend of the signal intensity between the 10th and 20th second of drying, but rather discuss the impact of solubility on drying. Possible reasons for a PL enhancement at this point in drying are phase segregation between polymer and fullerene molecules (i.e., fewer excitons are dissociated into charge carriers), solvent mixture effects (i.e., one solvent evaporates faster than the other), and polymer agglomeration. To understand this period of the PL signal during the initial drying phase, we measured the drying behavior of pristine DPP-TT-T in $o$-xylene only, at RT (Fig. S9, ESI $\dagger$ ). Interestingly, pristine DPP-TT-T in $o$-xylene showed a similar pattern, indicating that the 2nd period of drying is correlated with a specific DPP-TT-T behavior in $o$-xylene only. To further examine and understand this phenomenon, we investigated the solubility of DPP-TT-T. The very first signal of crystal formation was seen around the 20th second, indicating that the supersaturation and nucleation processes occurred before the 20th second, corresponding to the interval between the 10th and 20th seconds (Fig. 3c-e). This result was confirmed by measuring the solubility of DPP-TT-T in the $o$-xylene:mesitylene mixture at $60{ }^{\circ} \mathrm{C}$, which was approximately $8 \mathrm{mg} \mathrm{mL} \mathrm{m}^{-1}$ (13th second in Fig. 3c). The data indicate that, within this time period (between the 10th and 20th seconds of drying), the solution crosses the solubility limit, and reaches critical supersaturation and polymer chains start to form compact nucleates due to ongoing concentration enhancement caused by drying. As crystallization takes place at the 20th second, PL experiences a sharp decrease due to aggregation quenching.

In order to confirm the effect of solubility on the DPP-TT-T: PCBM PL signal, a series of in situ PL measurements of pristine DPP-TT-T were conducted at room temperature at concentrations of $3.75,5,7.5$ and $15 \mathrm{mg} \mathrm{mL}^{-1}$ (Fig. 4). These measurements were intentionally conducted at room temperature and without any PCBM in order to fully resolve DPP-TT-T behavior in the solution without additional quenching effects. Its PL behavior at lower concentrations ( 3.75 and $5 \mathrm{mg} \mathrm{mL}^{-1}$ ) was similar to that of the blend solution, confirming the fact that the signal behavior during the 10-20 second interval (region 2, Fig. 3c) is indeed not a temperature or PCBM effect, but a concentration effect. The solubility of DPP-TT-T at room temperature was determined as $6.5 \mathrm{mg} \mathrm{mL}^{-1}$, which supports the idea that the slightly increasing behavior of the PL signal starts right after the solubility limit is reached (Fig. 4). As expected, the drying kinetics of the $7.5 \mathrm{mg} \mathrm{mL}^{-1}$ solution did not exhibit this 2nd drying phase, as the concentration was above the
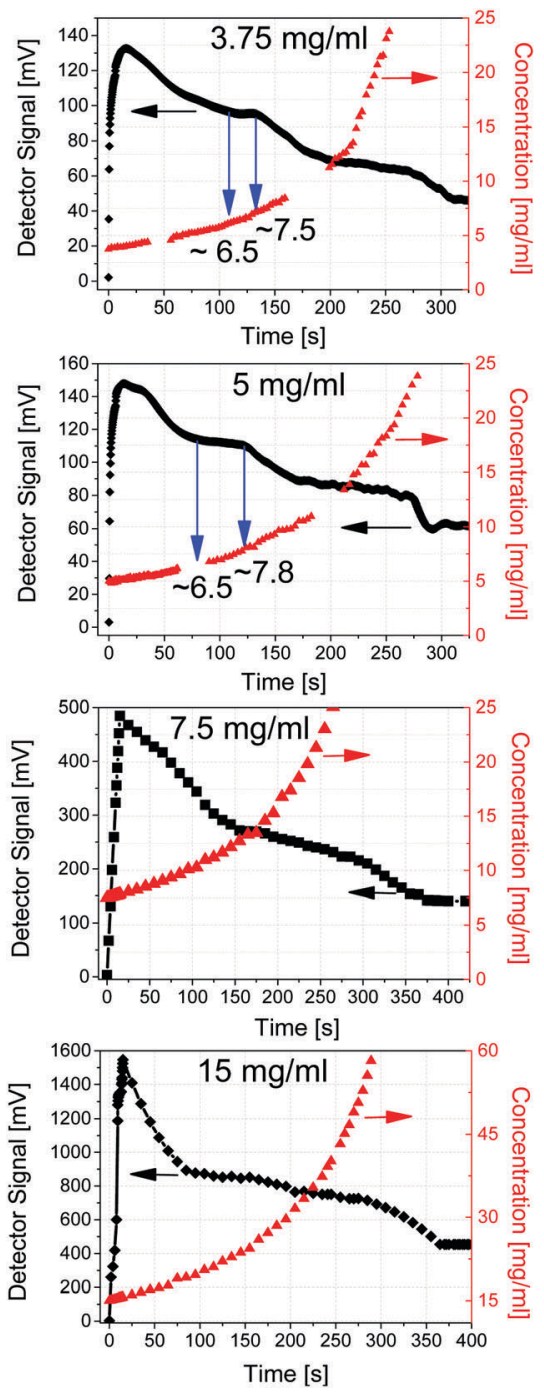

Fig. 4 Concentration dependent in situ PL measurements on pristine DPPTT-T solutions at concentrations of $3.75,5,7.5$ and $15 \mathrm{mg} \mathrm{mL}^{-1}$ at room temperature. Vertical arrows and values in panels indicate the concentration of the corresponding PL data. Horizontal arrows show the corresponding $y$-axis for each plot.

solubility limit from the beginning. In fact, the drying kinetics of a $15 \mathrm{mg} \mathrm{mL}^{-1}$ solution also resembled the pattern seen with the $3.75,5$ and $7.5 \mathrm{mg} \mathrm{mL}^{-1}$ solutions after the concentration passed the critical solubility limit. Additionally, this behaviour was also observed for pristine DPP-TT-T in $o$-xylene, as mentioned above (Fig. S9, ESI $\dagger$ ). The critical solubility limit of DPP-TT-T in $o$-xylene at RT was determined as $9.5 \mathrm{mg} \mathrm{mL}^{-1}$, which corresponds to the starting point of this 2nd drying phase approximately (Fig. S9, ESI $\dagger$ ). Hence, it was observed that DPP-TT-T underwent gelation in $o$-xylene after the solubility limit. If the gel aggregates remain relatively apart and free of PCBM, the diffusion-limited ordering could become inhibited since a polymer strand has only restricted freedom to move within a gel aggregate. This could explain the fact that the crystal coherence length and alkyl-chain packing remain constant with $8.5 \mathrm{~nm}$ and $22.7 \AA$ A, respectively (Fig. 3d and e). 


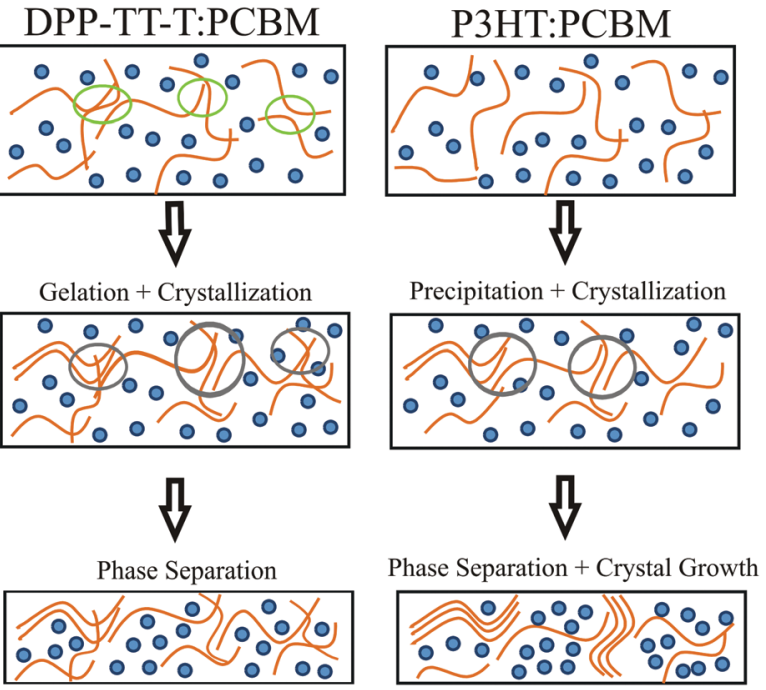

Fig. 5 Proposed model describing film formation during the drying process. Small blue circles and orange lines represent PCBM and the polymer, respectively. The DPP-TT-T:PCBM solution contains loose aggregates, indicated with large open circles in the top left panel. Gelation causes cross-linking of DPP-TT-T chains, indicated with large grey circles in the middle left panel. Large grey circles in the middle right panel indicate the ordering of $\mathrm{P} 3 \mathrm{HT}$ chains.

After the gelation period, DPP-TT-T gel aggregates collapsed to precipitates (solid-state formation) causing the last PL signal drop (region 3 in Fig. 3c). Completely dried DPP-TT-T formed weak crystal signals with edge-on (h00) series, at $q_{\mathrm{z}}$ positions of $0.23,0.51$ and $0.75 \AA^{-1}$, respectively. Due to the weak crystal signals, the background signal including X-ray window signals dominated most of the features, including the (010) peak (Fig. S6b, ESI †).

Two drying mechanisms measured very precisely with our drying chamber are summarized in Fig. 5. Due to gelation, DPP-TT-T:PCBM followed a very different path, compared to P3HT:PCBM. Gelation caused cross-linking of DPP-TT-T chains, hindering further ordering while precipitation and residual solvent in the P3HT:PCBM film enforced further organization. Additionally, both systems experience solid-liquid phase separation. While fullerene aggregates were the main cause for the DPP-TT-T:PCBM system, polymer-rich and fullerene-rich aggregates in the P3HT:PCBM film contribute to the measured LS signal.

\section{Conclusions}

In summary, we presented a flexible and portable in situ drying chamber, which is a powerful tool to completely characterize film formation of any organic, inorganic or hybrid thin film coating, without any need for additional extraneous measurements. As the chamber allows very precise and reproducible drying series (Fig. S10, ESI $\dagger$ ), the results presented on P3HT:PCBM and DPP-TT-T:PCBM blends represent the direct observation of the interplay between thermodynamics and kinetics, including phase separation, gelation and crystallization, and give deep insight into drying and microstructure formation mechanisms. In situ PL measurements, which have not been reported in previous drying studies, are proved to be especially powerful, as we were able to investigate the solution behavior in detail and correlate this behavior with drying effects. Using our novel in situ drying chamber, we will systematically investigate the drying mechanisms of multiple systems under several drying conditions to build a road map for solution formulation and morphology control in printed electronics, which can be directly adapted to large-scale processing.

\section{Acknowledgements}

Nusret S. Güldal and Thaer Kassar contributed equally to this work. The authors gratefully thank Deutsche Forschungsgemienschaft (DFG) in the framework of SFB 953 (Synthetic Carbon Allotropes) and Cluster of Excellence 'Engineering of Advanced Materials', Solar Technologies go Hybrid (SolTech) and Umweltfreundliche Hocheffiziente Organische Solarzellen (UOS) for their financial support and Solvay for providing DPP-TT-T in the framework of the X10D EU project. The authors specially thank Christian Bär for the setup construction. The authors acknowledge the European Synchrotron Radiation Facility (ESRF) for procurement of synchrotron radiation facilities.

\section{Notes and references}

1 F. C. Krebs, Sol. Energy Mater. Sol. Cells, 2009, 93, 394-412.

2 P. Kopola, T. Aernouts, S. Guillerez, H. Jin, M. Tuomikoski, A. Maaninen and J. Hast, Sol. Energy Mater. Sol. Cells, 2010, 94, 1673-1680.

3 L. Chang, I. E. Jacobs, M. P. Augustine and A. J. Moulé, Org. Electron., 2013, 14, 2431-2443.

4 R. Hegde, N. Henry, B. Whittle, H. Zang, B. Hu, J. Chen, K. Xiao and M. Dadmun, Sol. Energy Mater. Sol. Cells, 2012, 107, 112-124.

5 C. Koerner, C. Elschner, N. C. Miller, R. Fitzner, F. Selzer, E. Reinold, P. Bäuerle, M. F. Toney, M. D. McGehee, K. Leo and M. Riede, Org. Electron., 2012, 13, 623-631.

6 S. Kouijzer, J. J. Michels, M. van den Berg, V. S. Gevaerts, M. Turbiez, M. M. Wienk and R. A. J. Janssen, J. Am. Chem. Soc., 2013, 135, 12057-12067.

7 L. J. Richter, D. M. DeLongchamp, F. A. Bokel, S. Engmann, K. W. Chou, A. Amassian, E. Schaible and A. Hexemer, Adv. Energy Mater., 2015, 5, 1400975.

8 B. Schmidt-Hansberg, M. F. G. Klein, K. Peters, F. Buss, J. Pfeifer, S. Walheim, A. Colsmann, U. Lemmer, P. Scharfer and W. Schabel, J. Appl. Phys., 2009, 106, 124501.

9 A. J. Pearson, T. Wang and D. G. Lidzey, Rep. Prog. Phys, 2013, 76, 022501.

10 S. Y. Heriot and R. A. L. Jones, Nat. Mater., 2005, 4, 782-786.

11 B. Schmidt-Hansberg, M. Baunach, J. Krenn, S. Walheim, U. Lemmer, P. Scharfer and W. Schabel, Chem. Eng. Process., 2011, 50, 509-515. 
12 M. Sanyal, B. Schmidt-Hansberg, M. F. G. Klein, A. Colsmann, C. Munuera, A. Vorobiev, U. Lemmer, W. Schabel, H. Dosch and E. Barrena, Adv. Energy Mater., 2011, 1, 363-367.

13 B. Schmidt-Hansberg, M. Sanyal, M. F. G. Klein, M. Pfaff, N. Schnabel, S. Jaiser, A. Vorobiev, E. Müller, A. Colsmann, P. Scharfer, D. Gerthsen, U. Lemmer, E. Barrena and W. Schabel, ACS Nano, 2011, 5, 8579-8590.

14 B. Schmidt-Hansberg, M. Sanyal, N. Grossiord, Y. Galagan, M. Baunach, M. F. G. Klein, A. Colsmann, P. Scharfer, U. Lemmer, H. Dosch, J. Michels, E. Barrena and W. Schabel, Sol. Energy Mater. Sol. Cells, 2012, 96, 195-201.

15 B. Schmidt-Hansberg, M. F. G. Klein, M. Sanyal, F. Buss, G. Q. G. De Medeiros, C. Munuera, A. Vorobiev, A. Colsmann, P. Scharfer, U. Lemmer, E. Barrena and W. Schabel, Macromolecules, 2012, 45, 7948-7955.

16 K. W. Chou, B. Yan, R. Li, E. Q. Li, K. Zhao, D. H. Anjum, S. Alvarez, R. Gassaway, A. Biocca, S. T. Thoroddsen, A. Hexemer and A. Amassian, Adv. Mater., 2013, 25, 1923-1929.

17 J. Bergqvist, S. A. Mauger, K. Tvingstedt, H. Arwin and O. Inganäs, Sol. Energy Mater. Sol. Cells, 2013, 114, 89-98.

18 J. J. van Franeker, M. Turbiez, W. Li, M. M. Wienk and R. A. J. Janssen, Nat. Commun., 2015, 6, 6229.

19 M. Campoy-Quiles, T. Ferenczi, T. Agostinelli, P. G. Etchegoin, Y. Kim, T. D. Anthopoulos, P. N. Stavrinou, D. D. C. Bradley and J. Nelson, Nat. Mater., 2008, 7, 158-164.

20 T. Wang, A. D. F. Dunbar, P. A. Staniec, A. J. Pearson, P. E. Hopkinson, J. E. MacDonald, S. Lilliu, C. Pizzey, N. J. Terrill, A. M. Donald, A. J. Ryan, R. A. L. Jones and D. G. Lidzey, Soft Matter, 2010, 6, 4128.

21 A. J. Pearson, T. Wang, A. D. F. Dunbar, H. Yi, D. C. Watters, D. M. Coles, P. A. Staniec, A. Iraqi, R. A. L. Jones and D. G. Lidzey, Adv. Funct. Mater., 2014, 24, 659-667.

22 D. M. Smilgies, R. Li, G. Giri, K. W. Chou, Y. Diao, Z. Bao and A. Amassian, Phys. Status Solidi RRL, 2013, 7, 177-179.
23 F. Liu, W. Zhao, J. R. Tumbleston, C. Wang, Y. Gu, D. Wang, A. L. Briseno, H. Ade and T. P. Russell, Adv. Energy Mater., 2014, 4, 1-9.

24 M. Abdelsamie, K. Zhao, M. R. Niazi, K. W. Chou and A. Amassian, J. Mater. Chem. C, 2014, 2, 3373.

25 N. Shin, L. J. Richter, A. A. Herzing, R. J. Kline and D. M. DeLongchamp, Adv. Energy Mater., 2013, 3, 938-948.

26 K. Oku, S. Inasawa, Y. Tsuji and Y. Yamaguchi, Drying Technol., 2012, 30, 832-838.

27 R. Rodríguez, S. Jiménez-Sandoval, M. Estévez and S. Vargas, J. Non-Cryst. Solids, 2008, 354, 3623-3629.

28 F. MacHui, S. Langner, X. Zhu, S. Abbott and C. J. Brabec, Sol. Energy Mater. Sol. Cells, 2012, 100, 138-146.

29 P. Müller-Buschbaum, Adv. Mater., 2014, 26, 7692-7709.

30 R. Swanepoel, J. Phys. E., 1983, 16, 1214-1222.

31 S. Nilsson, A. Bernasik, A. Budkowski and E. Moons, Macromolecules, 2007, 40, 8291-8301.

32 T.-Q. Nguyen, I. B. Martini, J. Liu and B. J. Schwartz, J. Phys. Chem. B, 2000, 104, 237-255.

33 J. R. Lakowicz, Principles of Fluorescence Spectroscopy, Springer, New York City, 2006.

34 I. Meager, R. S. Ashraf, S. Mollinger, B. C. Schroeder, H. Bronstein, D. Beatrup, M. S. Vezie, T. Kirchartz, A. Salleo, J. Nelson and I. McCulloch, J. Am. Chem. Soc., 2013, 135, 11537-11540.

35 H. Wang, Y. Xu, X. Yu, R. Xing, J. Liu and Y. Han, J. Polym., 2013, 5, 1272-1324.

36 H. Bronstein, Z. Chen, R. S. Ashraf, W. Zhang, J. Du, J. R. Durrant, P. Shakya Tuladhar, K. Song, S. E. Watkins, Y. Geerts, M. M. Wienk, R. A. J. Janssen, T. Anthopoulos, H. Sirringhaus, M. Heeney and I. McCulloch, J. Am. Chem. Soc., 2011, 133, 3272-3275.

37 Z. Huang, E. C. Fregoso, S. Dimitrov, P. S. Tuladhar, Y. W. Soon, H. Bronstein, I. Meager, W. Zhang, I. McCulloch and J. R. Durrant, J. Mater. Chem. A, 2014, 2, 19282-19289.

38 R. D. Deegan, O. Bakajin, T. F. Dupont, G. Huber, S. R. Nagel and T. A. Witten, Phys. Rev. E: Stat. Phys., Plasmas, Fluids, Relat. Interdiscip. Top., 2000, 62, 756-765. 